\title{
ASYMPTOTIC EXPANSION OF THE BERGMAN KERNEL FOR TUBE DOMAIN OF INFINITE TYPE
}

\author{
HANJIN LEE
}

\begin{abstract}
The asymptotic expansions of the Bergman kernels on the diagonals near the boundary points of exponentially-flat infinite type for pseudoconvex tube domain in $\mathbb{C}^{2}$ are obtained.
\end{abstract}

\section{Introduction}

The studies of boundary behavior of Bergman kernel have been important topic for a long time related to the analysis of holomorphic functions for pseudoconvex domains. The boundary geometries of the domains determine the growth rates of the Bergman kernels near the given boundary points. For strongly pseudoconvex domain in $\mathbb{C}^{n}$, the boundary behavior of Bergman kernel is well understood by the works of Diederich [5], Hörmander [8] and Fefferman [7]. In particular, Fefferman obtained the asymptotic expansion formula for the Bergman kernel on the diagonal near $C^{\infty}$ smooth strongly pseudoconvex boundary point. For the weakly pseudoconvex domain of finite type in the sense of D'Angelo, there have been considerable and significant achievements. To cite only a few, we list here only $[2,3,4,6,14]$. In particular, Kamimoto obtained asymptotic expansions for the domains which have circular symmetries $[10]$ and for finite type tube domains $[9,11]$.

However, there are very few results for infinite type boundary points. Kim and Lee [12] analyzed Bergman kernel near the exponentially-flat infinite type boundary point for model domain using scaling method. Bharali [1] obtained upper and lower bound estimates of the Bergman kernel near the mildly infinite type boundary point for model domain.

In this paper we obtain an asymptotic expansion of the Bergman kernel function $B(z, \bar{z})$ for any point $z$ near an exponentially-flat infinite type boundary point $z_{0}$ of a pseudoconvex tube domain in $\mathbb{C}^{2}$.

We use Koranyi-Vinberg integral representation of the Bergman kernel for tube domain. Since the singularity of the Bergman kernel near given boundary point is determined by local geometry of the boundary, analysis is focused on

Received August 8, 2011.

2010 Mathematics Subject Classification. Primary 32A25; Secondary 32A07.

Key words and phrases. Bergman kernel, infinite type, tube domains. 
localized representation of original Koranyi-Vinberg representation. As in the case of finite type, singularity can be stratified by a real blowing up in the case of infinite type.

Since the principal part of defining function for finite type case gives quasihomogeneous model, the analysis of the Bergman kernel near finite type boundary point focuses on how to handle a perturbation of model case. On the other hand, we don't have such a model for infinite type case. For this reason, first of all, a real blowing up of the infinite type point is constructed using defining function itself. In addition, a proper flat condition for the defining function is needed to analyze the principal part of singularity expansion. Our condition controls the growth of derivatives of all order of the defining function at the infinite type boundary point such that the principal part of expansion formula extends to the horizontal boundary in stratification. Since $f(t)=\exp \left(\frac{1}{t^{2 m}}\right)$ satisfies that condition, we call our condition exponentially-flat. Under this growth condition, it will be shown that all the coefficients of expansion in vertical direction vanish.

\section{Statement of main theorem}

Let $\Omega$ be a domain in $\mathbb{C}^{2}$. Denote by $A^{2}(\Omega)$ the closed subspace of $L^{2}(\Omega)$ consisting of holomorphic functions. Take a complete orthonormal basis $\left\{\varphi_{j}\right\}_{j}$ of $A^{2}(\Omega)$. The Bergman kernel $B(z)=B(z, \bar{z})$ of $\Omega$ (on the diagonal) is defined by $B(z)=\sum_{j}\left|\varphi_{j}(z)\right|^{2}$.

Given a domain $\omega$ in $\mathbb{R}^{2}$, the tube domain over the base $\omega$ is defined by

$$
\Omega=\mathbb{R}^{2}+i \omega=\left\{z=x+i y \in \mathbb{C}^{2}: x \in \mathbb{R}^{2}, y \in \omega\right\} .
$$

Here we set $x=\left(x_{1}, x_{2}\right), y=\left(y_{1}, y_{2}\right), z=\left(z_{1}, z_{2}\right)$. It is known that $\Omega$ is pseudoconvex if and only if $\omega$ is convex. We assume that $\omega$ is convex with $C^{\infty}$-smooth boundary. Suppose the boundary point $z_{0}=x_{0}+i y_{0}$ is of infinite type. That is, there exists a coordinate system $\left(y, V_{y_{0}}\right)$ such that

$$
\omega \cap V_{y_{0}}=\left\{\left(y_{1}, y_{2}\right) \in \mathbb{R}^{2}: y_{2}>f\left(y_{1}\right)\right\},
$$

where $f$ is nonnegative, convex and $C^{\infty}$-smooth, satisfies $f(0)=0$ and vanishes to infinite order at $y_{1}=0$, that is, $\lim _{t \rightarrow 0} f(t) /|t|^{N}=0$ for each $N \in \mathbb{Z}_{+}$.

In this article, we will use the notation $A(t) \sim B(t)$ in place of $\lim _{t \rightarrow 0+}\left|\frac{A(t)}{B(t)}\right|$ is finite and nonzero constant. For positive valued function $A(t)$ and $B(t)$, we will also use the notation $A(t) \lesssim B(t)$ if there exists $C(t)$ which is positive constant or satisfies $\lim _{t \rightarrow 0+} C(t)>0$ such that $A(t) \leq C(t) B(t)$ near $t=0$.

We say that the boundary point $z_{0} \in \partial \Omega$ is exponentially-flat, if there exists a coordinate system such as (2.1) such that

$$
\frac{f^{(k)}(t)}{f(t)} \sim t^{-(2 m+1) k} .
$$

In particular, $f(t)=\exp \left(-\frac{1}{t^{2 m}}\right)$ satisfies exponentially-flat condition. 
We say that the boundary point $z_{0}$ is quasi-symmetric if the defining function $f$ additionally satisfies following: if there exist positive constants $d$ and $\epsilon$ such that $f(-t)=f(d t)$ for $0<t<\epsilon$.

Consider a real blowing up of the origin for quasi-symmetric function $f$ as follows: $\pi:(-1 / d, 1) \times(0, \epsilon) \rightarrow \omega \cap\left\{\left(y_{1}, y_{2}\right): y_{2}<L\right\}$ for some positive constant $L$ such that $\pi(\tau, \rho)=\left(y_{1}, y_{2}\right)$, where $y_{1}=\tau \rho, y_{2}=f(\rho)$. Then the main theorem of this article is as follows:

Theorem 2.1. Let $z_{0}=x_{0}+i y_{0}$ be a non Levi-flat, exponentially-flat infinite type and quasi-symmetric boundary point of $\Omega$. There exists a neighborhood $V$ of $y_{0}$ and $\epsilon$ such that for $(\tau, \rho) \in(-1 / d, 1) \times(0, \epsilon) \subset \pi^{-1}(V \cap \omega)$ and $x$ near $x_{0}$

$$
B(x+i \pi(\tau, \rho))=\rho^{-2} f(\rho)^{-2} B_{0}(\tau, \rho), \quad(\bmod \text { smooth kernel) }
$$

where $B_{0}(\tau, \rho)$ is a $C^{\infty}$ smooth function on $(-1 / d, 1) \times(0, \epsilon)$ satisfies followings:

(1) $B_{0}(\tau, \rho)$ extends $C^{\infty}$ smoothly to $(-1 / d, 1) \times[0, \epsilon)$.

(2) $B_{0}(\tau, \rho)$ has the following asymptotic expansion with respect to $\rho:$ for each $N \geq 1$, there exists $R_{N}(\tau, \rho) \in C((-1 / d, 1) \times[0, \epsilon))$ such that

$$
B_{0}(\tau, \rho)=b(\tau)+R_{N}(\tau, \rho) \rho^{N}
$$

$$
\begin{array}{r}
\text { on }(-1 / d, 1) \times(0, \epsilon) \text { where } b(\tau) \text { is in } C^{\infty}(-1 / d, 1) \text { and } \\
\left|R_{N}(\tau, \rho)\right| \lesssim \rho^{-(2 m+2) N-3} f(\rho) \quad \text { as } \rho \rightarrow 0+.
\end{array}
$$

Remark 1. The principal term $b(\tau)$ tells us that tangential limits of Bergman kernel depend on orbits approaching to the origin. Each vertical line $\{\tau\} \times$ $\left(0, \rho_{0}\right)$ is mapped by $\pi$ to $\left\{y_{2}=f\left(y_{1} / \tau\right): 0<y_{1}<\tau \rho_{0}\right\}$, which is a tangential orbit to the origin. Then

$$
\begin{aligned}
b(\tau) & =\lim _{\rho \rightarrow 0+} B_{0}(\tau, \rho) \\
& =\lim _{\rho \rightarrow 0+} \frac{1}{2 \pi^{2}} \int_{0}^{\infty} e^{-2 s}\left[\int_{-a \rho / f(\rho)}^{a \rho / f(\rho)} e^{-2 \tau s v} \frac{1}{\int_{-\epsilon / \rho}^{\epsilon / \rho} e^{-2 s[v w+f(\rho w) / f(\rho)] d w}}\right] s^{2} d s
\end{aligned}
$$

for $-1 / d<\tau<1$.

Remark 2. Our theorem still holds for infinite type points which become flat less rapidly than exponentially-flat case. The defining function

$$
f(t)=\exp \left(-\frac{1}{t^{2 m}} \log \frac{1}{|t|}\right)
$$

becomes flat less rapidly than exponentially-flat case. It holds that for any positive constant $\alpha$ there exists a neighborhood $U_{\alpha}$ of 0 such that $\left|\frac{f^{(k)}(t)}{f(t)}\right| \lesssim$ $t^{-(2 m+1) k-\alpha}$ whenever $t \in U_{\alpha}$. All the estimates still work under this growth condition with minor change. 


\section{Integral formula for tube domain}

For $y=\left(y_{1}, y_{2}\right), u=\left(u_{1}, u_{2}\right)$ in $\mathbb{R}^{2}$, we set $\langle y, u\rangle=y_{1} u_{1}+y_{2} u_{2}$. It is shown in [13], [15] that the Bergman kernel of a tube domain $\Omega=\mathbb{R}^{2}+i \omega$ is expressed as

$$
B(z)=B(x+i y)=\frac{1}{4 \pi^{2}} \int_{\Lambda^{*}} e^{-2\langle y, u\rangle} \frac{1}{\varphi(u)} d u
$$

where

$$
\varphi(u)=\int_{\omega} e^{-2\langle u, w\rangle} d w
$$

and $\Lambda^{*}=\left\{u \in \mathbb{R}^{2}: \varphi(u)<\infty\right\}$. We need to express $\Lambda^{*}$ in a different form to compute the Bergman kernel. For a convex set $\omega$ we define its recession cone

$$
\Lambda_{\omega}=\left\{y \in \mathbb{R}^{2}: v+t y \in \omega \text { for all } v \in \omega, t \geq 0\right\} .
$$

Then for unbounded $\omega$, we have

$$
\Lambda^{*}=\left\{u \in \mathbb{R}^{2}:\langle u, y\rangle>0 \text { for } y \in \Lambda_{\omega}\right\} .
$$

For $R>0$, set $\tilde{B}_{R}=\left\{y_{1} \in \mathbb{R}: f\left(y_{1}\right)<R\right\}, B_{R}=\left\{y_{1} / R: y_{1} \in \tilde{B}_{R}\right\}$ and $B=\cap_{R>0} B_{R}$. Then

$$
\Lambda_{\omega}=\left\{\left(s \hat{y}_{1}, s\right) \in \mathbb{R}^{2}: s \geq 0, \hat{y}_{1} \in B\right\} .
$$

Thus we have

$$
\Lambda^{*}=\left\{\left(t \hat{u}_{1}, t\right) \in \mathbb{R}^{2}: t>0, \hat{u}_{1} \in B^{*}\right\}
$$

where

$$
B^{*}=\left\{\hat{u}_{1} \in \mathbb{R}: \hat{u}_{1} \hat{y}_{1}+1>0 \text { for } \hat{y}_{1} \in B\right\} .
$$

For our case $B^{*}=\mathbb{R}$, thus

$$
\begin{aligned}
& B(x+i y)=\frac{1}{2 \pi^{2}} \int_{0}^{\infty} e^{-2 y_{2} t} F\left(y_{1}, t\right) t^{2} d t \\
& F\left(y_{1}, t\right)=\int_{\mathbb{R}} e^{-2 t y_{1} \hat{u}_{1}} \frac{1}{D\left(t, \hat{u}_{1}\right)} d \hat{u}_{1}, \\
& D\left(t, \hat{u}_{1}\right)=\int_{\mathbb{R}} e^{-2 t\left[f(w)+\hat{u}_{1} w\right]} d w .
\end{aligned}
$$

\section{Localization}

Localization for integral representation of the Bergman kernel allows us to analyze its singularity using only local geometry around infinite type boundary point. Localization of Bergman kernel for tube domain were introduced in [9], [11]. We follow the arguments in [11]. Let $U$ be an open interval containing the origin. Then we define

where

$$
B_{U}(z)=\frac{1}{4 \pi^{2}} \int_{\Lambda^{*}(U)} e^{-2\langle y, u\rangle} \frac{1}{\varphi(u)} d u,
$$

$$
\Lambda^{*}(U):=\left\{\left(t \hat{u}_{1}, t\right) \in \mathbb{R}^{2}: t>0, \hat{u}_{1} \in U\right\}
$$


and

where

$$
B_{U, \epsilon}(z)=\frac{1}{4 \pi^{2}} \int_{\Lambda^{*}(U)} e^{-2\langle y, u\rangle} \frac{1}{\varphi_{\epsilon}(u)} d u
$$

$$
\varphi_{\epsilon}(u)=\int_{\omega \cap[(-\epsilon, \epsilon) \times \mathbb{R}+]} e^{-2\langle u, w\rangle} d w .
$$

The following localization arguments hold if the defining function $f$ of $\omega$ satisfies $f(t)=o(|t|)$ as $t \rightarrow 0$.

Proposition 4.1 (Kamimoto). For any open interval $U$ containing the origin, $B(z)-B_{U}(z)$ is real analytic near the origin.

Proposition 4.2 (Kamimoto). For any $\epsilon>0$, there exists $U$ such that $B_{U}(z)-$ $B_{U, \epsilon}(z)$ is real analytic near the origin.

Thus, for our purposes, it suffices to obtain the asymptotic expansion of $B_{U, \epsilon}(z)$.

\section{Proof of main theorem}

The localized representation of the Bergman kernel is as follows:

$$
\begin{gathered}
B_{a, \epsilon}\left(y_{1}, y_{2}\right)=\frac{1}{2 \pi^{2}} \int_{0}^{\infty} e^{-2 y_{2} t} F_{a, \epsilon}\left(y_{1} ; t\right) t^{2} d t \\
F_{a, \epsilon}\left(y_{1} ; t\right)=\int_{-a}^{a} e^{-2 t y_{1} u} \frac{1}{D_{\epsilon}(t, u)} d u \\
D_{\epsilon}(t, u)=\int_{-\epsilon}^{\epsilon} e^{-2 t[u w+f(w)]} d w
\end{gathered}
$$

where $a$ depends on the choice of $\epsilon$ according to Proposition 4.2. By the blow-up coordinate system $\pi(\tau, \rho)=(\tau \rho, f(\rho)),(\tau, \rho) \in(-1 / d, 1) \times(0, \epsilon)$ the representation in $(3.2)$ changes into

$$
\begin{aligned}
& B_{a, \epsilon}(\Phi(\tau, \rho))=\rho^{-2} f(\rho)^{-2} K_{a, \epsilon}(\tau, \rho), \\
& K_{a, \epsilon}(\tau, \rho)=\frac{1}{2 \pi^{2}} \int_{0}^{\infty} e^{-2 s} s^{2} F_{a, \epsilon}(\tau, \rho ; s) d s, \\
& F_{a, \epsilon}(\tau, \rho ; s)=\int_{-\mu}^{\mu} e^{-2 \tau s v} \frac{1}{D_{\epsilon}(\rho ; s, v)} d v \\
& D_{\epsilon}(\rho ; s, v)=\int_{-\epsilon / \rho}^{\epsilon / \rho} e^{-2 s[v w+f(\rho w) / f(\rho)]} d w,
\end{aligned}
$$

where $\mu=a \rho / f(\rho)$.

We decompose the integral $K_{a, \epsilon}$ of (5.1) as follows:

$$
\begin{aligned}
K_{a, \epsilon}(\tau, \rho) & =\frac{1}{2 \pi^{2}} \int_{f(\rho)}^{\infty} e^{-2 s} F_{a, \epsilon}(\tau, \rho ; s) s^{2} d s+\frac{1}{2 \pi^{2}} \int_{0}^{f(\rho)} e^{-2 s} F_{a, \epsilon}(\tau, \rho ; s) s^{2} d s \\
& =K_{a, \epsilon}^{(1)}(\tau, \rho)+K_{a, \epsilon}^{(2)}(\tau, \rho) .
\end{aligned}
$$


We will show that boundary values of derivatives of $K_{a, \epsilon}^{(1)}(\tau, \rho)$ at $\rho=0$ equal 0 in Proposition 5.1 and $\rho^{-2} f(\rho)^{-2} K_{a, \epsilon}^{(2)}(\tau, \rho)$ is real analytic in $\rho$ and $f(\rho)$ in Proposition 5.2. It will prove the main theorem.

\subsection{Growth estimate of derivative of $K_{a, \epsilon}^{(1)}$}

In this section we will use the notation $\partial_{\rho}^{n}$ in place of $\frac{\partial^{n}}{\partial \rho^{n}}$ whenever we need to simplify expression.

Proposition 5.1. Suppose that $f$ satisfies exponentially flat condition (2.2) at the origin. Then

$$
\left|\partial_{\tau}^{n^{\prime}} \partial_{\rho}^{n} K_{a, \epsilon}^{(1)}(\tau, \rho)\right| \lesssim \rho^{-(2 m+2) n-3} f(\rho) \quad \text { as } \rho \rightarrow 0+
$$

for $-1 / d<\tau<1$ and $n \geq 1, n^{\prime} \geq 0$.

Proof. We have

$$
\begin{aligned}
& \partial_{\tau}^{n^{\prime}} \partial_{\rho}^{n} K_{a, \epsilon}^{(1)}(\tau, \rho) \\
= & -\sum_{k=0}^{n-1} \partial_{\rho}^{n-1-k}\left(\left.f^{\prime}(\rho) f(\rho)^{2} e^{-2 f(\rho)} \partial_{\tau}^{n^{\prime}} \partial_{\rho}^{k} F_{a, \epsilon}(\tau, \rho ; s)\right|_{s=f(\rho)}\right) \\
& +\frac{1}{2 \pi^{2}} \int_{f(\rho)}^{\infty} e^{-2 s} \partial_{\tau}^{n^{\prime}} \partial_{\rho}^{n} F_{a, \epsilon}(\tau, \rho ; s) s^{2} d s
\end{aligned}
$$

for $n \geq 1$. The first part of (5.2) is estimated as follows. Set

$$
\begin{aligned}
F_{a, \epsilon}^{(k)}(\tau, \rho ; s) & =\int_{-\mu}^{\mu} e^{-2 \tau s v} \partial_{\rho}^{k} \frac{1}{D_{\epsilon}(\rho ; s, v)} d v \\
Q_{j}^{+}(\tau, \rho ; s) & =\left.\mu^{\prime} e^{-2 \tau s \mu} \partial_{\rho}^{j} \frac{1}{D_{\epsilon}(\rho ; s, v)}\right|_{v=\mu}, \\
Q_{j}^{-}(\tau, \rho ; s) & =\left.\mu^{\prime} e^{2 \tau s \mu} \partial_{\rho}^{j} \frac{1}{D_{\epsilon}(\rho ; s, v)}\right|_{v=-\mu} .
\end{aligned}
$$

Then we have

$$
\partial_{s}^{i} \partial_{\tau}^{n^{\prime}} \partial_{\rho}^{k} F_{a, \epsilon}(\tau, \rho ; s)=\sum_{j=0}^{k-1} \partial_{s}^{i} \partial_{\tau}^{n^{\prime}} \partial_{\rho}^{k-1-j}\left(Q_{j}^{+}+Q_{j}^{-}\right)+\partial_{s}^{i} \partial_{\tau}^{n^{\prime}} F_{a, \epsilon}^{(k)}, \quad k \geq 1 .
$$

By Lemma 6.6, we have

$$
\left.\partial_{s}^{i} \partial_{\tau}^{n^{\prime}} \partial_{\rho}^{k-1-j} Q_{j}^{ \pm}(\tau, \rho ; s)\right|_{s=f(\rho)} \sim \rho^{-(2 m+1) k+n^{\prime}+1} f(\rho)^{-i-1} .
$$

By Lemma 6.5, we have

$$
\begin{aligned}
& \left.\partial_{s}^{i} \partial_{\tau}^{n^{\prime}} F_{a, \epsilon}^{(k)}(\tau, \rho ; s)\right|_{s=f(\rho)} \\
= & \left.\sum_{i_{1}+i_{2}=i} C_{i_{1}, i_{2}} \tau^{i_{1}}(-f(\rho))^{n^{\prime}} \int_{-\mu}^{\mu} e^{-2 \tau s v} v^{n^{\prime}+i_{1}} \partial_{s}^{i_{2}} \partial_{\rho}^{k} \frac{1}{D_{\epsilon}} d v\right|_{s=f(\rho)} \\
\sim & \rho^{-(2 m+1) k+n^{\prime}+2} f(\rho)^{-i-1} .
\end{aligned}
$$


They imply

$$
\left.\left(\partial_{s}^{i} \partial_{\tau}^{n^{\prime}} \partial_{\rho}^{k} F_{a, \epsilon}\right)\right|_{s=f(\rho)} \sim \rho^{-(2 m+1) k+n^{\prime}+1} f(\rho)^{-i-1} .
$$

Using (5.6) and derivative condition on $f$ we have

$$
\begin{aligned}
& \partial_{\rho}^{j}\left(\left.\left(\partial_{\tau}^{n^{\prime}} \partial_{\rho}^{k} F_{a, \epsilon}\right)\right|_{s=f(\rho)}\right) \\
= & \left.\left(\partial_{\tau}^{n^{\prime}} \partial_{\rho}^{k+j} F_{a, \epsilon}\right)\right|_{s=f(\rho)} \\
& +\left.\sum_{j^{\prime}=0}^{j-1} \sum_{j^{\prime \prime}=1}^{j-j^{\prime}}\left(\partial_{s}^{j^{\prime \prime}} \partial_{\tau}^{n^{\prime}} \partial_{\rho}^{k+j^{\prime}} F_{a, \epsilon}\right)\right|_{s=f(\rho)} \sum_{\substack{\alpha_{1}+\cdots+\alpha_{j^{\prime \prime}=j-j^{\prime}} \\
\alpha_{1}, \ldots, \alpha_{j^{\prime \prime}}>0}} f^{\left(\alpha_{1}\right) \cdots f^{\left(\alpha_{j^{\prime \prime}}\right)}} \\
(5.7) \sim & \rho^{-(2 m+1)(k+j)+n^{\prime}+1} f(\rho)^{-1} .
\end{aligned}
$$

By

$$
\begin{aligned}
& \partial_{\rho}^{j}\left(f^{\prime}(\rho) f(\rho)^{2} e^{-2 f(\rho)}\right) \\
= & e^{-f(\rho)} \sum_{j^{\prime}=0}^{j} \sum_{\alpha_{1}+\cdots+\alpha_{j^{\prime}+3}=j+1} C_{\alpha_{1}, \ldots, \alpha_{j^{\prime}+3}} f^{\left(\alpha_{1}\right)}(\rho) \cdots f^{\left(\alpha_{j^{\prime}+3}\right)}(\rho) \\
\sim & \rho^{-(2 m+1)(j+1)} f(\rho)^{3}
\end{aligned}
$$

and (5.7) we have

$$
\sum_{k=0}^{n-1} \partial_{\rho}^{n-1-k}\left(\left.f^{\prime}(\rho) f(\rho)^{2} e^{-f(\rho)} \partial_{\tau}^{n^{\prime}} \partial_{\rho}^{k} F_{a, \epsilon}(\tau, \rho ; s)\right|_{s=f(\rho)}\right) \sim \rho^{-(2 m+1) n+n^{\prime}+1} f(\rho)^{2} .
$$

The second part of (5.2) is separated by (5.5) as

$$
\begin{aligned}
& \int_{f(\rho)}^{\infty} e^{-2 s} \partial_{\tau}^{n^{\prime}} \partial_{\rho}^{n} F_{a, \epsilon}(\tau, \rho ; s) s^{2} d s \\
= & \sum_{j=0}^{n-1} \int_{f(\rho)}^{\infty} e^{-2 s} \partial_{\tau}^{n^{\prime}} \partial_{\rho}^{n-1-j}\left(Q_{j}^{+}+Q_{j}^{-}\right)(\tau, \rho ; s) s^{2} d s \\
& +\int_{f(\rho)}^{\infty} e^{-2 s} \partial_{\tau}^{n^{\prime}} F_{a, \epsilon}^{(n)}(\tau, \rho ; s) s^{2} d s .
\end{aligned}
$$

The first part of (5.9) is estimated by following lemma.

Lemma 5.1. Under the assumptions of Proposition 5.1,

$$
\left|\int_{f(\rho)}^{\infty} e^{-2 s} \partial_{\tau}^{n^{\prime}} \partial_{\rho}^{n-1-j} Q_{j}^{ \pm}(\tau, \rho ; s) s^{2} d s\right| \lesssim \rho^{-(2 m+2)(n-1)-2} f(\rho)^{2}
$$

for $j=0,1, \ldots, n-1$. 
Proof. Estimation of integration of derivatives of (5.4) requires a revised equation of (6.16) which is not evaluated at $s=f(\rho)$. Applying Lemma 6.7 to the first line of (6.16), we have

$$
\begin{aligned}
\left.\partial_{\rho}^{q}\left(\left.\partial_{\rho}^{j} \frac{1}{D_{\epsilon}}\right|_{v=\mu}\right) \sim \rho^{-(2 m+1)(q+j)} \frac{1}{D_{\epsilon}}\right|_{v=\mu}\left(\sum_{j^{\prime}=1}^{q} \sum_{j^{\prime \prime}=1}^{j^{\prime}} \sum_{k=0}^{q+j-j^{\prime}} C_{j^{\prime}, j^{\prime \prime}, k}^{(q, j)}(\rho ; s) s^{j^{\prime \prime}+k} f^{-j^{\prime \prime}-k}\right. \\
\left.+\sum_{k=0}^{q+j} C_{k}^{(q, j)}(\rho ; s) s^{k} f^{-k}\right),
\end{aligned}
$$

where $C_{j^{\prime}, j^{\prime \prime}, k}^{(q, j)}(\rho ; s)=O(1)$ and $C_{k}^{(q, j)}(\rho ; s)=O(1)$ as $\rho \rightarrow 0+$. Applying it and (6.15) into (6.14), we have

$$
\begin{aligned}
& \partial_{\tau}^{n^{\prime}} \partial_{\rho}^{n-1-j} Q_{j}^{ \pm}(\tau, \rho ; s) \\
\sim & \left.\sum_{q_{1}+q_{2}=n-1-j} C_{q_{1}, q_{2}} \rho^{-(2 m+1)(n-1)+n^{\prime}+1} f^{-n^{\prime}-1} s^{n^{\prime}} e^{\mp \mu \tau s} \frac{1}{D_{\epsilon}}\right|_{v= \pm \mu} \\
& \sum_{r=0}^{q_{1}}(-\tau)^{r} \rho^{r}\left(\sum_{j^{\prime}=1}^{q_{2}} \sum_{j^{\prime \prime}=1}^{j^{\prime}} \sum_{k=0}^{q_{2}+j-j^{\prime}} C_{j^{\prime}, j^{\prime \prime}, k}^{\left(q_{2}, j\right)}(\rho ; s) s^{j^{\prime \prime}+k+r} f^{-j^{\prime \prime}-k-r}+\sum_{k=0}^{q_{2}+j} C_{k}^{\left(q_{2}, j\right)}(\rho ; s) s^{k+r} f^{-k-r}\right) .
\end{aligned}
$$

Set

$$
f_{0}(t)=\max \{f(t), f(-t)\} \quad \text { for } 0<t<\epsilon .
$$

It holds that

$\left.D_{\epsilon}(\rho ; s, v)\right|_{v=\mu} \geq \int_{-\epsilon}^{\epsilon} e^{-2 s(\mu w+f(\rho w) / f(\rho))} d w$

$$
\begin{aligned}
& \gtrsim e^{-2 s f_{0}(\epsilon \rho) / f(\rho)} \frac{\sinh (2 \epsilon \mu s)}{\mu s} \\
& \gtrsim e^{2\left(-f_{0}(\epsilon \rho) / f(\rho)+\epsilon \mu\right) s} \frac{\left(1-e^{-4 a \epsilon \rho}\right)}{\rho} f(\rho) s^{-1} \quad \text { for } s \geq f(\rho) .
\end{aligned}
$$

It implies that

$$
\begin{aligned}
& \quad\left|\int_{f(\rho)}^{\infty} e^{-2 s} \partial_{\tau}^{n^{\prime}} \partial_{\rho}^{n-1-j} Q_{j}^{ \pm} s^{2} d s\right| \\
& \lesssim \sum_{q_{1}+q_{2}=n-1-j} C_{q_{1}, q_{2}} \rho^{-(2 m+1)(n-1)+n^{\prime}+1} f(\rho)^{-n^{\prime}-1} \sum_{r=0}^{q_{1}}|\tau|^{r} \rho^{r} \\
& \quad \int_{f(\rho)}^{\infty} e^{-\lambda s}\left(\sum_{j^{\prime}=1}^{q_{2}} \sum_{j^{\prime \prime}=1}^{j^{\prime}} \sum_{k=0}^{q_{2}+j-j^{\prime}} f(\rho)^{-j^{\prime \prime}-k-r} s^{j^{\prime \prime}+k+r+n^{\prime}+2}+\sum_{k=0}^{q_{2}+j} f(\rho)^{-k-r} s^{k+r+n^{\prime}+2}\right) d s \\
& \sim \rho^{-(2 m+2)(n-1)-2} f(\rho)^{2}, \\
& \text { where } \lambda=1-\frac{f_{0}(\epsilon \rho)}{f(\rho)}+\mu(|\tau|+\epsilon) . \text { It proves the lemma. }
\end{aligned}
$$

The boundary limit of the second integral of (5.9) is estimated as follows. 
Lemma 5.2. Under the assumptions of Proposition 5.1,

$$
\left|\int_{f(\rho)}^{\infty} e^{-2 s} \partial_{\tau}^{n^{\prime}} F_{a, \epsilon}^{(n)}(\tau, \rho ; s) s^{2} d s\right| \lesssim \rho^{-(2 m+2) n-2} f(\rho)^{2}
$$

for $-1 / d<\tau<1, n \geq 1$.

Proof. By Lemma 6.4 there exist bounded functions $E_{l}^{(p)}(\rho ; s, v) \in C\left(\left[0, \rho_{0}\right) \times\right.$ $\left.\mathbb{R}_{+} \times \mathbb{R}\right)$ such that

$$
\begin{aligned}
& \partial_{\tau}^{n^{\prime}} F_{a, \epsilon}^{(n)}(\tau, \rho ; s) \\
= & \int_{-\mu}^{\mu} e^{-2 \tau s v}(-s v)^{n^{\prime}} \partial_{\rho}^{n} \frac{1}{D_{\epsilon}(\rho ; s, v)} d v \\
\sim & \sum_{p=0}^{n} \sum_{l=0}^{p} \rho^{-n-l-N_{l}} f(\rho)^{-p+l} s^{p+n^{\prime}} \int_{-\mu}^{\mu} e^{-2 \tau s v} E_{l}^{(p)}(\rho ; s, v) \frac{1}{D_{\epsilon}(\rho ; s, v)} v^{l+n^{\prime}} d v,
\end{aligned}
$$

where $N_{p}=0$ and $N_{l}=2 m(n-l)$ for $l<p$. Using (5.10) we have

$$
\begin{aligned}
& \left|\int_{-\mu}^{\mu} e^{-2 \tau s v} E_{l}^{(p)}(\rho ; s, v) \frac{1}{D_{\epsilon}(\rho ; s, v)} v^{l+n^{\prime}} d v\right| \\
\leq & \int_{0}^{\mu}\left(e^{-2(\epsilon+|\tau|) s v}+e^{-2(\epsilon-|\tau|) s v}\right) \frac{2 s v e^{2 s f_{0}(\rho \epsilon) / f(\rho)}}{1-e^{-2 \epsilon s v}} v^{l+n^{\prime}} d v .
\end{aligned}
$$

It holds that

$$
\begin{aligned}
& \int_{0}^{\mu} e^{-2(\epsilon \pm|\tau|) s v} \frac{2 s v}{1-e^{-2 \epsilon s v}} v^{l+n^{\prime}} d v \\
\leq & \frac{e^{2 a \epsilon \rho}}{\epsilon} \int_{0}^{\frac{a \rho}{2 s}} e^{-(2 \epsilon \pm|\tau|) s v} v^{l+n^{\prime}} d v+C(\rho) \rho^{-1} s \int_{\frac{a \rho}{2 s}}^{\mu} e^{-2(\epsilon \pm|\tau|) s v} v^{l+n^{\prime}+1} d v \\
\lesssim & \frac{\left(1 / s+\rho^{-1} \mu\right)}{(\epsilon \pm|\tau|)} \mu^{l+n^{\prime}} e^{-(\epsilon \pm|\tau|) \mu s} \sinh ((\epsilon \pm|\tau|) \mu s),
\end{aligned}
$$

where $C(\rho) \rightarrow C>0$ as $\rho \rightarrow 0+$. Thus

$$
\begin{aligned}
& \int_{f(\rho)}^{\infty} e^{-2 s\left(1-f_{0}(\epsilon \rho) / f(\rho)\right)} s^{p+n^{\prime}+2}\left[\int_{0}^{\mu} e^{-2(\epsilon \pm|\tau|) s v} \frac{2 s v}{1-e^{-2 \epsilon s v}} v^{l+n^{\prime}} d v\right] d s \\
\lesssim & \frac{\sinh \left(\frac{\epsilon \pm|\tau|}{2}\right)}{\epsilon \pm|\tau|} \rho^{-p+l-3} f(\rho)^{p-l+2}
\end{aligned}
$$

for $|\tau|<\epsilon$. It implies that for $|\tau|<\epsilon$

$$
\left|\int_{f(\rho)}^{\infty} e^{-2 s} F_{n}(\tau, \rho ; s) s^{2} d s\right| \lesssim \sum_{p=0}^{n} \sum_{l=0}^{p} \rho^{-n-p-N_{l}-3} f(\rho)^{2} \sim \rho^{-(2 m+2) n-3} f(\rho)^{2} .
$$


For $|\tau| \geq \epsilon$, by $D_{\epsilon}(\rho ; s, v) \gtrsim e^{-s f_{0}(\epsilon \rho) / f(\rho)}$

$$
\begin{aligned}
& \int_{f(\rho)}^{\infty} e^{-2 s} s^{p+n^{\prime}+2}\left[\int_{-\mu}^{\mu} e^{-2|\tau| s v} \frac{1}{D_{\epsilon}(\rho ; s, v)} v^{l+n^{\prime}} d v\right] d s \\
\leq & \int_{f(\rho)}^{\infty} e^{-2\left(1-\frac{f_{0}(\epsilon \rho)}{f(\rho)}-\mu|\tau|\right) s} s^{p+n^{\prime}+2} \mu^{l+n^{\prime}+1} d s \\
\sim & \rho^{-p+l-2} f(\rho)^{p-l+2} .
\end{aligned}
$$

Thus for $|\tau| \geq \epsilon$,

$$
\begin{aligned}
& \left|\int_{f(\rho)}^{\infty} e^{-2 s} \partial_{\tau}^{n^{\prime}} F_{n}(\tau, \rho ; s) s^{2} d s\right| \\
\lesssim & \sum_{p=0}^{n} \sum_{l=0}^{p} \rho^{-n-p-N_{l}-2} f(\rho)^{2} \sim \rho^{-(2 m+2) n-2} f(\rho)^{2} .
\end{aligned}
$$

It proves the lemma.

Now by (5.8), Lemma 5.1 and Lemma 5.2, we obtain Proposition 5.1.

\subsection{Real analyticity of $K_{2}$}

Proposition 5.2. $\rho^{-2} f(\rho)^{-2} K_{2}(\tau, \rho)$ is real analytic in $\rho$ and $f(\rho)$.

Proof. Set $F_{j}(\tau, \rho)=\left.\frac{1}{j !} \partial_{s}^{j} F_{a, \epsilon}(\tau, \rho ; s)\right|_{s=0}$. Then

$$
K_{2}(\tau, \rho)=\sum_{j=0}^{N-1} F_{j}(\tau, \rho) \int_{0}^{f(\rho)} e^{-2 s} s^{j+2} d s+\int_{0}^{f(\rho)} e^{-2 s} F_{N}(\tau, \rho ; s) s^{N+2} d s .
$$

We have

$$
\begin{aligned}
F_{j}(\tau, \rho) & =\frac{1}{j !} \sum_{j_{1}+j_{2}=j}\left(\begin{array}{c}
j \\
j_{1}
\end{array}\right)(-\tau)^{j_{1}} \sum_{k=0}^{j_{2}} C_{j_{2}, k} \rho^{-k+1} f(\rho)^{-j_{2}+k} \int_{-\mu}^{\mu} v^{j_{1}+k} d v \\
& =\rho^{2} f(\rho)^{-j-1} \sum_{j^{\prime}=0}^{j} C_{j, j^{\prime}}^{\prime}(\tau \rho)^{j^{\prime}}
\end{aligned}
$$

and

$$
\begin{aligned}
& \sup _{0 \leq s \leq f(\rho)}\left|F_{N}(\tau, \rho ; s)\right| \\
\leq & \frac{1}{N !} \sum_{j_{1}+j_{2}=N}\left(\begin{array}{l}
N \\
j_{1}
\end{array}\right)|\tau|^{j_{1}} \sum_{k=0}^{j_{2}} \rho^{-k} f(\rho)^{-j_{2}+k} \sup _{0 \leq s \leq f(\rho)} \int_{-\mu}^{\mu} e^{-\tau s v}|v|^{j_{1}+k}\left|\tilde{D}_{k}^{\left(j_{2}\right)}(\rho ; s, v)\right| \frac{1}{D_{\epsilon}} d v \\
\leq & C_{\epsilon} e^{|\tau| \rho} \rho^{2} f(\rho)^{-N-1} \sum_{j^{\prime}=0}^{N} C^{\prime \prime}{ }_{N, j^{\prime}}(|\tau| \rho)^{j^{\prime}}
\end{aligned}
$$

where $\tilde{D}_{k}^{\left(j_{2}\right)}$ is bounded for $v \in \mathbb{R}$. In particular, $C^{\prime \prime}{ }_{N, j} \leq M^{N}$ for $0 \leq j \leq N$ for some positive number $M$. It completes the proof. 


\section{Proof of lemmas}

In this section we will give proofs of lemmas which we use in the proof of main proposition.

\subsection{Estimates of derivatives of $D_{\epsilon}$ and $\frac{1}{D_{\epsilon}}$}

Here we present estimates of growths of derivatives of $D_{\epsilon}$, which we need to estimate $\partial_{\rho}^{n} \partial_{\tau}^{n^{\prime}} \partial_{\rho}^{n} K_{a, \epsilon}^{(1)}$ through (5.1) and (5.2). The firsthand estimate is on growth of derivatives of $\frac{1}{D_{\epsilon}(\rho ; s, v)}$ and its evaluation at $v=\mu$ or $s=f(\rho)$. We have

$$
D_{\epsilon}(\rho ; s, v)=\int_{-\epsilon / \rho}^{\epsilon / \rho} e^{-2 s\left(v w+\frac{f(\rho w)}{f(\rho)}\right)} d w=\rho^{-1} \int_{-\epsilon}^{\epsilon} e^{-2 s\left(\frac{v u}{\rho}+\frac{f(u)}{f(\rho)}\right)} d u .
$$

Set $\varphi(u, v ; \rho)=\frac{v u}{\rho}+\frac{f(u)}{f(\rho)}$ for convenience.

Lemma 6.1. For $q \geq 1$,

$$
\begin{aligned}
& \partial_{v}^{p} \partial_{\rho}^{q} \partial_{s}^{r} D_{\epsilon}(\rho ; s, v) \\
\sim & \rho^{-p-q-1} \sum_{r^{\prime}=\max (r-p, 0)}^{r} s^{p-r+r^{\prime}} \int_{-\epsilon}^{\epsilon} e^{-s \varphi} \varphi^{r^{\prime}} u^{p} d u \\
& +\sum_{q^{\prime}=1}^{q} \rho^{-p-q+q^{\prime}-1} \sum_{j=1}^{q^{\prime}} \sum_{r^{\prime}=\max (r-p-j, 0)}^{r} \sum_{|\beta|=q^{\prime}} s^{p+j-r+r^{\prime}} \int_{-\epsilon}^{\epsilon} e^{-s \varphi} \varphi^{r^{\prime}} \partial_{\rho}^{\beta} \varphi u^{p} d u,
\end{aligned}
$$

where $|\beta|=\beta_{1}+\cdots+\beta_{j}$ and $\partial_{\rho}^{\beta} \varphi=\partial_{\rho}^{\beta_{1}} \varphi \cdots \partial_{\rho}^{\beta_{j}} \varphi$.

Proof. We have

$$
\begin{aligned}
& \partial_{v}^{p} \partial_{\rho}^{q} \partial_{s}^{r} D_{\epsilon} \\
= & \partial_{\rho}^{q} \partial_{s}^{r}\left[(-s)^{p} \rho^{-p-1} \int_{-\epsilon}^{\epsilon} e^{-s \varphi} u^{p} d u\right] \\
= & C_{p, q} \rho^{-p-q-1} \int_{-\epsilon}^{\epsilon} \partial_{s}^{r}\left((-s)^{p} e^{-s \varphi}\right) u^{p} d u \\
& +\sum_{q^{\prime}=1}^{q} \sum_{j=1}^{q^{\prime}} \sum_{|\beta|=q^{\prime}} C_{p, q, q^{\prime}, \beta} \rho^{-p-q+q^{\prime}-1} \int_{-\epsilon}^{\epsilon} \partial_{s}^{r}\left((-s)^{p+j} e^{-s \varphi}\right) \partial_{\rho}^{\beta} \varphi u^{p} d u .
\end{aligned}
$$

Using

$$
\partial_{s}^{r}\left(s^{p} e^{-s \varphi}\right)=\sum_{r^{\prime}=\max (r-p, 0)}^{r} C_{r, r^{\prime}} s^{p-r+r^{\prime}} \varphi^{r^{\prime}} e^{-s \varphi}
$$

we obtain the lemma. 
Lemma 6.2. Suppose that $f$ satisfies exponentially-flat condition (2.2) at the origin. Then

$$
\left.\partial_{v}^{p} \partial_{\rho}^{q} \partial_{s}^{r} \frac{1}{D_{\epsilon}}\right|_{v=\mu, s=f(\rho)} \sim \rho^{-p-(2 m+1) q-1} f(\rho)^{p-r} \quad \text { as } \rho \rightarrow 0+.
$$

Proof. For $\beta_{1}+\cdots+\beta_{j}=q^{\prime}$,

$$
\left.\partial_{\rho}^{\beta_{1}} \varphi \cdots \partial_{\rho}^{\beta_{j}} \varphi\right|_{v=\mu} \sim \rho^{-(2 m+1) q^{\prime}} f(\rho)^{-j}\left(f(u)^{j}+\Psi_{q^{\prime}, j}(\rho, u)\right),
$$

where $\Psi_{q^{\prime}, j}(\rho, u)=O(\rho)$ as $\rho \rightarrow 0+$. By Lemma 6.1

$$
\begin{aligned}
& \left.\partial_{v}^{p} \partial_{\rho}^{q} \partial_{s}^{r} D_{\epsilon}\right|_{v=\mu, s=f(\rho)} \\
\sim & \rho^{-p-q-1} f(\rho)^{p-r} \int_{-\epsilon}^{\epsilon} e^{-s \varphi} \sum_{l=0}^{\min (p, r)}(a u+f(u))^{l} u^{p} d u \\
& +\sum_{q^{\prime}=1}^{q} \rho^{-p-q-2 m q^{\prime}-1} f(\rho)^{p-r} \sum_{j=1}^{q^{\prime}} \int_{-\epsilon}^{\epsilon} e^{-s \varphi} u^{p}\left(f(u)^{j}+\Psi_{q^{\prime \prime}, j}(\rho, u)\right) \sum_{l=0}^{\min (p, r)}(a u+f(u))^{l} d u \\
\sim & \rho^{-p-(2 m+1) q-1} f(\rho)^{p-r} .
\end{aligned}
$$

Thus we have

$$
\begin{aligned}
& \left.\partial_{v}^{p} \partial_{\rho}^{q} \partial_{s}^{r} \frac{1}{D_{\epsilon}}\right|_{v=\mu, s=f(\rho)} \\
= & \sum_{l=1}^{\max (p, q, r)} \sum_{\substack{|\alpha|=p,|\beta|=q,|\gamma|=r \\
\sim}} C_{\alpha, \beta, \gamma} \frac{\partial_{v}^{\alpha_{1}} \partial_{\rho}^{\beta_{1}} \partial_{s}^{\gamma_{1}} D_{\epsilon}}{D_{\epsilon}} \cdots \frac{\partial_{v}^{\alpha_{l}} \partial_{\rho}^{\beta_{l}} \partial_{s}^{\gamma_{l}} D_{\epsilon}}{D_{\epsilon}} \frac{1}{D_{\epsilon}} \\
& \sum^{-p-(2 m+1) q} f(\rho)^{p-r} .
\end{aligned}
$$

We obtain the lemma.

For such growth estimates of evaluations of the derivatives of $D_{\epsilon}$ as Lemma $6.2,(6.1)$ is enough. But if we want to estimate integral of derivatives of $\frac{1}{D_{\epsilon}}$, we need more thorough estimate than (6.1). Set $D_{p, q}(\rho ; s, v)=\int_{-\epsilon}^{\epsilon} e^{-s \varphi} u^{p} f(u)^{q} d u$.

Lemma 6.3. Suppose that $f$ satisfies exponentially flat condition (2.2) at the origin. Then

$$
\begin{gathered}
\sum_{\beta_{1}+\cdots+\beta_{j}=q} \int_{-\epsilon}^{\epsilon} e^{-s \varphi} \partial_{\rho}^{\beta_{1}} \varphi \cdots \partial_{\rho}^{\beta_{j}} \varphi d u \\
\sim \sum_{l=0}^{j} v^{l} \rho^{-q-l-2 m(q-l)\left(1-\delta_{j-l, 0}\right)} f(\rho)^{-j+l} D_{l, j-l}(\rho ; s, v) .
\end{gathered}
$$

Proof. We have

$$
\partial_{\rho}^{\beta_{1}} \varphi \cdots \partial_{\rho}^{\beta_{j}} \varphi=\sum_{l=0}^{j} v^{j-l} u^{j-l} f(u)^{l} \rho^{-j+l} H_{(\beta, l)}(\rho),
$$


where

$$
H_{(\beta, l)}(\rho)=\sum_{\left(\beta_{1}^{\prime}, \ldots, \beta_{j}^{\prime}\right)} C_{\beta_{1}^{\prime}, \ldots, \beta_{j}^{\prime}}\left(\frac{1}{f(\rho)}\right)^{\left(\beta_{1}^{\prime}\right)} \cdots\left(\frac{1}{f(\rho)}\right)^{\left(\beta_{l}^{\prime}\right)} \rho^{-\left(\beta_{l+1}^{\prime}+\cdots+\beta_{j}^{\prime}\right)},
$$

where $\left(\beta_{1}^{\prime}, \ldots, \beta_{j}^{\prime}\right)$ is a rearrangement of $\left(\beta_{1}, \ldots, \beta_{j}\right)$ and summation is taken over all possible such rearrangements. Then since $\left(\frac{1}{f(\rho)}\right)^{(p)} \sim \rho^{-(2 m+1) p} f(\rho)^{-1}$,

$$
\begin{aligned}
H_{(\beta, l)} & \sim f(\rho)^{-l} \sum_{\left(\beta_{1}^{\prime}, \ldots, \beta_{j}^{\prime}\right)} C_{\beta_{1}^{\prime}, \ldots, \beta_{j}^{\prime}} \rho^{-(2 m+1)\left(\beta_{1}^{\prime}+\cdots+\beta_{l}^{\prime}\right)-\left(\beta_{l+1}^{\prime}+\cdots+\beta_{j}^{\prime}\right)} \\
& \sim f(\rho)^{-l} \sum_{\left(\beta_{1}^{\prime}, \ldots, \beta_{j}^{\prime}\right)} C_{\beta_{1}^{\prime}, \ldots, \beta_{j}^{\prime}} \rho^{-2 m\left(\beta_{1}^{\prime}+\cdots+\beta_{l}^{\prime}\right)-q} \\
& \sim \begin{cases}\rho^{-q} & l=0 \\
\rho^{-q-2 m(q-j+l)} f(\rho)^{-l} & l \geq 1 .\end{cases}
\end{aligned}
$$

It proves the lemma.

Lemma 6.4. Under the same assumption of Lemma 6.3, for $n \geq 1$, there exists $E_{l}^{(p)}(\rho ; s, v) \in C\left(\left[0, \rho_{0}\right) \times \mathbb{R}_{+} \times \mathbb{R}\right)$ satisfying $E_{l}^{(p)}(\rho ; s, v) \lesssim 1$ such that

$$
\partial_{\rho}^{n} \frac{1}{D_{\epsilon}(\rho ; s, v)} \sim \sum_{p=0}^{n} \sum_{l=0}^{p} \rho^{-n-l-N_{l}} f(\rho)^{-p+l} s^{p} \frac{E_{l}^{p}(\rho ; s, v)}{D_{\epsilon}(\rho ; s, v)} v^{l},
$$

where $N_{p}=0$ and $N_{l}=2 m(n-l)$ for $l<p$.

Proof. Set $\hat{D}_{p, q}=D_{p, q} / D_{\epsilon}$. Then by Lemmas 6.1 and 6.3 , we have

$$
\begin{aligned}
\frac{\partial_{\rho}^{q} D_{\epsilon}}{D_{\epsilon}} & =\rho^{-q}+\sum_{\substack{q^{\prime}+q^{\prime \prime}=q \\
q^{\prime \prime} \geq 1}} \rho^{-q^{\prime}-1} \sum_{j=1}^{q^{\prime \prime}}(-s)^{j} \sum_{\beta_{1}+\cdots+\beta_{j}=q^{\prime \prime}} \frac{1}{D_{\epsilon}} \int_{-\epsilon}^{\epsilon} e^{-s \varphi} \partial_{\rho}^{\beta_{1}} \varphi \cdots \partial_{\rho}^{\beta_{j}} \varphi d u \\
& \sim \rho^{-q}\left(1+\sum_{j=1}^{q}\left(\sum_{l=0}^{j} B_{j, l}^{(q)}(\rho ; s, v) v^{l}\right)(-s)^{j}\right),
\end{aligned}
$$

where

$$
B_{j, l}^{(q)}(\rho ; s, v)= \begin{cases}\rho^{-j} \hat{D}_{j, 0} & l=j \\ \rho^{-2 m q+(2 m-1) l} f^{-j+l} \hat{D}_{l, j-l} & 0 \leq l \leq j-1 .\end{cases}
$$

We have

$$
\begin{aligned}
\partial_{\rho}^{n} \frac{1}{D_{\epsilon}} & =\sum_{k=1}^{n} \sum_{\substack{\alpha_{1}+\cdots, \alpha_{k}=n \\
\alpha_{1}, \ldots, \alpha_{k}>0}} \frac{\partial_{\rho}^{\alpha_{1}} D_{\epsilon}}{D_{\epsilon}} \cdots \frac{\partial_{\rho}^{\alpha_{k}} D_{\epsilon}}{D_{\epsilon}} \frac{1}{D_{\epsilon}} \\
& \sim \rho^{-n} \sum_{p=0}^{n}(-s)^{p} \sum_{l=0}^{p} \hat{B}_{l}^{(p)}(\rho ; s, v) v^{l} \frac{1}{D_{\epsilon}}
\end{aligned}
$$


where

$$
\hat{B}_{l}^{(p)}(\rho ; s, v)=\sum_{k=1}^{n} \sum_{\substack{\alpha_{1}+\cdots+\alpha_{k}=n \\ \alpha_{1}, \ldots, \alpha_{k}>0}} \sum_{j_{1}+\cdots+j_{k}=p} \sum_{\substack{l_{1}+\cdots+l_{k}=l \\ l_{1} \leq j_{1}, \ldots, l_{k} \leq j_{k}}} B_{j_{1}, l_{1}}^{\left(\alpha_{1}\right)} \cdots B_{j_{k}, l_{k}}^{\left(\alpha_{k}\right)} .
$$

Define

$$
E_{l}^{(p)}(\rho ; s, v)=\rho^{l+N_{l}} f(\rho)^{p-l} \hat{B}_{l}^{(p)}(\rho ; s, v),
$$

where $N_{p}=0$ and $N_{l}=m(n-l)$ for $l<p$. Then $E_{l}^{(p)} \lesssim 1$. It is proved as follows. For $l=p, \hat{B}_{l}^{(p)}$ is a sum of such terms as $B_{j_{1}, j_{1}}^{\left(\alpha_{1}\right)} \cdots B_{j_{k}, j_{k}}^{\left(\alpha_{k}\right)}=$ $\rho^{-p} \hat{D}_{j_{1}, 0} \cdots \hat{D}_{j_{k}, 0}$. For $l<p, \hat{B}_{l}^{(p)}$ is a sum of such terms as

$$
\begin{aligned}
& B_{j_{1}, j_{1}}^{\left(\alpha_{1}\right)} \cdots B_{j_{k^{\prime}}, j_{k^{\prime}}}^{\left(\alpha_{k}\right)} B_{j_{k^{\prime}+1}, l_{k^{\prime}+1}}^{\left(\alpha_{k}\right)} \cdots B_{j_{k}, l_{k}}^{\left(\alpha_{k}\right)} \\
= & \rho^{-\left(j_{1}+\cdots+j_{k^{\prime}}\right)-m\left(\alpha_{k^{\prime}+1}+\cdots+\alpha_{k}\right)+(m-1)\left(l_{k^{\prime}+1}+\cdots+l_{k}\right)} \\
& \hat{D}_{j_{1}, 0} \cdots \hat{D}_{j_{k^{\prime}}, 0} \cdots \hat{D}_{l_{k^{\prime}+1}, j_{k^{\prime}+1}-l_{k^{\prime}+1}} \cdots \hat{D}_{l_{k}, j_{k}-l_{k}},
\end{aligned}
$$

where $k^{\prime}$ satisfies $0<k^{\prime}<k$ and $l_{1}=j_{1}, \ldots, l_{k^{\prime}}=j_{k^{\prime}}, l_{k^{\prime}+1}<j_{k^{\prime}+1}, \ldots, l_{k}<$ $j_{k}$. Then the exponent of $\rho^{-1}$ is $l+m\left\{\left(\alpha_{k^{\prime}+1}-l_{k^{\prime}+1}\right)+\cdots+\left(\alpha_{k}-l_{k}\right)\right\}$. Since $\left(j_{k^{\prime}+1}-l_{k^{\prime}+1}\right)+\cdots+\left(j_{k}-l_{k}\right)=p-l$ and $\left(\alpha_{k^{\prime}+1}-j_{k^{\prime}+1}\right)+\cdots+\left(\alpha_{k}-j_{k}\right) \leq n-p$, the exponent of $\rho^{-1} \leq l-m(n-p+p-l)=l-m(n-l)$. The exponent of $f(\rho)^{-1}$ is $\left(j_{k^{\prime}+1}-l_{k^{\prime}+1}\right)+\cdots+\left(j_{k}-l_{k}\right)=p-l$. It completes the proof.

\subsection{Integrals of derivatives of $\frac{1}{D_{\epsilon}}$}

In this section we will estimate integrals in $v$ of derivatives of $\frac{1}{D_{\epsilon}(\rho ; s, v)}$. It require more thorough analysis than Lemma 6.2.

Lemma 6.5. For $q \geq 1$

$$
\left.\int_{-\mu}^{\mu} e^{-\tau s v} v^{n} \partial_{\rho}^{q} \partial_{s}^{r} \frac{1}{D_{\epsilon}(\rho ; s, v)} d v\right|_{s=f(\rho)} \sim \rho^{-(2 m+1) q+n+2} f(\rho)^{-r-n-1}
$$

as $\rho \rightarrow 0+$.

Proof. From Lemmas 6.1 and 6.3

$$
\begin{aligned}
& \left.\frac{\partial_{\rho}^{q} \partial_{s}^{r} D_{\epsilon}}{D_{\epsilon}}\right|_{s=f(\rho)} \\
\sim & \rho^{-q} \sum_{r^{\prime}=0}^{r} v^{r^{\prime}} \rho^{-r^{\prime}} f(\rho)^{r^{\prime}-r} \hat{D}_{r^{\prime}, r-r^{\prime}} \\
& +\rho^{-q} \sum_{q^{\prime}=1}^{q} \sum_{j=1}^{q^{\prime}} \sum_{l=0}^{j} \sum_{r^{\prime}=0}^{r} v^{l+r^{\prime}} \rho^{-\left(l+r^{\prime}\right)-2 m\left(q^{\prime}-l\right)\left(1-\delta_{l j}\right)} f(\rho)^{l+r^{\prime}-r} \hat{D}_{(j, l),\left(r, r^{\prime}\right)}
\end{aligned}
$$




$$
\sim \rho^{-q} \sum_{q^{\prime}=0}^{q} \sum_{l=0}^{q^{\prime}} \sum_{r^{\prime}=0}^{r} v^{l+r^{\prime}} \rho^{-\left(l+r^{\prime}\right)-2 m\left(q^{\prime}-l\right)} f(\rho)^{l+r^{\prime}-r} \hat{D}_{l,\left(r, r^{\prime}\right)}^{\left(q^{\prime}\right)},
$$

where

$$
\hat{D}_{(j, l),\left(r, r^{\prime}\right)}= \begin{cases}\sum_{r^{\prime \prime}=0}^{r-r^{\prime}} \hat{D}_{l+r^{\prime}, j-l+r^{\prime \prime}} & j \geq r \text { or } j<r, r^{\prime}>r-j \\ \sum_{r^{\prime \prime}=r-j-r^{\prime}}^{r-r^{\prime}}, \hat{D}_{l+r^{\prime}, j-l+r^{\prime \prime}} & j<r, 0 \leq r^{\prime} \leq r-j\end{cases}
$$

and

$$
\hat{D}_{l,\left(r, r^{\prime}\right)}^{\left(q^{\prime}\right)}= \begin{cases}\sum_{j=l+1}^{q^{\prime}} \hat{D}_{(j, l),\left(r, r^{\prime}\right)} & l<q^{\prime} \\ \sum_{j=1}^{q^{\prime}} \hat{D}_{(j, 0),\left(r, r^{\prime}\right)} & l=q^{\prime}>0 \\ \hat{D}_{(0,0),\left(r, r^{\prime}\right)} & l=q^{\prime}=0 .\end{cases}
$$

Set

$$
\text { (6.5) } G_{k}^{(q, r)}(\rho, f(\rho) ; v)=\sum_{\substack{l+r^{\prime}=k \\ 0 \leq l \leq q, 0 \leq r^{\prime} \leq r}} \sum_{q^{\prime}=0}^{q-l} \rho^{-2 m q^{\prime}} \hat{D}_{l,\left(r, r^{\prime}\right)}^{\left(l+q^{\prime}\right)}, \quad 0 \leq k \leq q+r .
$$

Then (6.4) has an expansion in $v$ as follows:

$$
\left.\frac{\partial_{\rho}^{q} \partial_{s}^{r} D_{\epsilon}}{D_{\epsilon}}\right|_{s=f(\rho)} \sim \sum_{k=0}^{q+r} v^{k} \rho^{-q-k} f(\rho)^{k-r} G_{k}^{(q, r)}(\rho, f(\rho) ; v) .
$$

From (6.6) we have

$$
\begin{aligned}
\left.\partial_{\rho}^{q} \partial_{s}^{r} \frac{1}{D_{\epsilon}}\right|_{s=f(\rho)} & \sim \sum_{i=1}^{\max (q, r)} \sum_{\substack{q_{1}+\cdots+q_{i}=q \\
r_{1}+\cdots+r_{i}=r}} \frac{\partial_{\rho}^{q_{1}} \partial_{s}^{r_{1}} D_{\epsilon}}{D_{\epsilon}} \cdots \frac{\partial_{\rho}^{q_{i}} \partial_{s}^{r_{i}} D_{\epsilon}}{D_{\epsilon}} \frac{1}{D_{\epsilon}} \\
& \sim \sum_{k=0}^{q+r} v^{k} \rho^{-q-k} f(\rho)^{k-r} A_{k}^{(q, r)}(\rho, f(\rho) ; v) \frac{1}{D_{\epsilon}}
\end{aligned}
$$

where

$$
A_{k}^{(q, r)}(\rho, f(\rho) ; v)=\sum_{i=1}^{\max (q, r)} \sum_{\substack{q_{1}+\cdots+q_{i}=q \\ r_{1}+\cdots+r_{i}=r}} \sum_{\substack{0_{1}+\cdots+k_{i} \leq q_{1}+r_{1}, \ldots, 0 \leq k_{i} \leq q_{i}+r_{i} \\ 0.0 .}} G_{k_{1}}^{\left(q_{1}, r_{1}\right)} \cdots G_{k_{i}}^{\left(q_{i}, r_{i}\right)}
$$

Since $\hat{D}_{l, l^{\prime}} \lesssim 1$ and $D_{\epsilon} \gtrsim \rho^{-1} e^{-s f(\epsilon) / f(\rho)}$, we have

$$
\left.\left|A_{k}^{(q, r)}(\rho, f(\rho) ; v) \frac{1}{D_{\epsilon}}\right|\right|_{s=f(\rho)} \lesssim\left\{\begin{array}{ll}
\rho^{-2 m q+1}, & k \leq r \\
\rho^{-2 m(q+r-k)+1}, & k>r
\end{array} \quad \text { as } \rho \rightarrow 0+.\right.
$$

Since

$$
\int_{-\epsilon}^{\epsilon} e^{-s \varphi} d u \leq \frac{2 \rho}{s v} \sinh (\epsilon s v / \rho) \lesssim e^{\epsilon s v / \rho}
$$


and

we have

$$
\left|D_{l, l^{\prime}}(\rho ; s, v)\right| \gtrsim \begin{cases}\frac{1}{\rho} e^{-s f(\epsilon) / f(\rho)}, & l: \text { even } \\ \frac{1}{\rho^{2}} s|v| e^{-s f(d \epsilon) / f(\rho)}, & l: \text { odd }\end{cases}
$$

By (6.5), (6.8) and (6.10), there exist nonzero integers $N_{i}, N_{i}^{\prime}$ such that

$$
\begin{aligned}
& \mid \begin{array}{ll}
A_{k}^{(q, r)}(\rho, f(\rho) ; v) \frac{1}{D_{\epsilon}} \mid & \left.\right|_{s=f(\rho)} \\
\sum_{i=1}^{\max (q, r)} \rho^{-2 m q+1}\left(\frac{f(\rho)}{\rho}|v|\right)^{N_{i}} e^{-\epsilon(i+1) \frac{f(\rho)}{\rho} v}, & k \leq r \\
\sum_{i=1}^{\max (q, r)} \rho^{-2 m(q+r-k)+1}\left(\frac{f(\rho)}{\rho}|v|\right)^{N_{i}^{\prime}} e^{-\epsilon(i+1) \frac{f(\rho)}{\rho} v}, & k>r
\end{array}
\end{aligned}
$$

as $\rho \rightarrow 0+$. Since

$$
\int_{-\mu}^{\mu} e^{-\tau f(\rho) v} v^{l} d v= \begin{cases}2 \rho^{l+1} f(\rho)^{-l-1} \sum_{k=0}^{\infty} \frac{\tau^{2 k} \rho^{2 k}}{(2 k) !(2 k+l+1)}, & l: \text { even } \\ -2 \rho^{l+2} f(\rho)^{-l-1} \sum_{k=0}^{\infty} \frac{\tau^{2 k+1} \rho^{2 k}}{(2 k+1) !(2 k+l+2)}, & l: \text { odd }\end{cases}
$$

we have

$\left.\int_{-\mu}^{\mu} e^{-\tau s v} v^{l} A_{k}^{(q, r)}(\rho, f(\rho) ; v) \frac{1}{D_{\epsilon}} d v\right|_{s=f(\rho)} \sim \begin{cases}\rho^{-2 m q+l+\delta_{l}} f(\rho)^{-l-1}, & k \leq r \\ \rho^{-2 m(q+r-k))+l+\delta_{l}} f(\rho)^{-l-1}, & k>r\end{cases}$ where $\delta_{l}=2$ if $l$ is even and $\delta_{l}=3$ if $l$ is odd. By applying (6.13) to (6.7), we obtain the lemma.

\subsection{Estimation of derivatives of $F_{a, \epsilon}(\tau, \rho ; s)$}

In the proof of Proposition 5.1, the main estimate of $\partial_{\tau}^{n^{\prime}} \partial_{\rho}^{n} K_{a, \epsilon}^{(1)}(\tau, \rho)$ is reduced to estimate of derivatives of $F_{a, \epsilon}(\tau, \rho ; s)$, which is decomposed into two parts by (5.5). Recall that the first part is given by the sum of

$$
\partial_{\rho}^{k-1-j} \partial_{\tau}^{n^{\prime}} Q_{j}^{+}(\tau, \rho ; s)=\partial_{\rho}^{k-1-j} \partial_{\tau}^{n^{\prime}}\left(\left.\mu^{\prime} e^{-\mu \tau s} \partial_{\rho}^{j} \frac{1}{D_{\epsilon}(\rho ; s, v)}\right|_{v=\mu}\right) .
$$

We will estimate of the growths of them evaluated at $s=f(\rho)$ and of its integrals in $s$.

Lemma 6.6. Suppose that $f$ satisfies exponentially flat condition (2.2) at the origin. Then

$$
\left.\partial_{s}^{p} \partial_{\rho}^{q} \partial_{\tau}^{n^{\prime}} Q_{j}^{ \pm}(\tau, \rho ; s)\right|_{s=f(\rho)} \sim \rho^{-(2 m+1)(q+j+1)+n^{\prime}+1} f(\rho)^{-p-1}
$$

as $\rho \rightarrow 0+$. 
Proof. We have

(6.14)

$$
\begin{aligned}
& \partial_{s}^{p} \partial_{\rho}^{q} \partial_{\tau}^{n^{\prime}} Q_{j}^{ \pm} \\
& \sim \sum_{\substack{p_{0}+p_{1}+p_{2}=p \\
q_{1}+q_{2}=q}} C{\substack{p_{0}, p_{1}, p_{2} \\
q_{1}, q_{2}}}(-s)^{n^{\prime}-p_{0}} \partial_{s}^{p_{1}} \partial_{\rho}^{q_{1}}\left(\mu^{\prime} \mu^{n^{\prime}} e^{\mp \mu \tau s}\right) \partial_{s}^{p_{2}} \partial_{\rho}^{q_{2}}\left(\left.\partial_{\rho}^{j} \frac{1}{D_{\epsilon}}\right|_{v= \pm \mu}\right) .
\end{aligned}
$$

Since

(6.15)

$$
\begin{aligned}
& \partial_{s}^{p_{1}} \partial_{\rho}^{q_{1}}\left(\mu^{\prime} \mu^{n^{\prime}} e^{-\mu \tau s}\right) \\
= & (-\tau)^{p_{1}}\left(\left(\mu^{\prime} \mu^{n^{\prime}+p_{1}}\right)^{\left(q_{1}\right)}+\sum_{l=1}^{q_{1}}\left(\mu^{\prime} \mu^{n^{\prime}+p_{1}}\right)^{\left(q_{1}-l\right)} \sum_{r=1}^{l} \sum_{|\beta|=l}(-\tau s)^{r} \mu^{\left(\beta_{1}\right)} \cdots \mu^{\left(\beta_{r}\right)}\right) e^{-\mu \tau s} \\
\sim & \sum_{r=0}^{q_{1}}(-\tau)^{p_{1}+r} s^{r} \rho^{-(2 m+1)\left(q_{1}+1\right)+n^{\prime}+p_{1}+r+1} f(\rho)^{-n^{\prime}-p_{1}-r-1} e^{-\mu \tau s}
\end{aligned}
$$

for $q_{1}>0$ and by Lemma 6.2

$$
\begin{aligned}
& \left.\partial_{s}^{p_{2}} \partial_{\rho}^{q_{2}}\left(\left.\partial_{\rho}^{j} \frac{1}{D_{\epsilon}}\right|_{v=\mu}\right)\right|_{s=f(\rho)} \\
= & \left.\sum_{j^{\prime}=1}^{q_{2}} \sum_{j^{\prime \prime}=1}^{j^{\prime}}\left(\partial_{s}^{p_{2}} \partial_{v}^{j^{\prime \prime}} \partial_{\rho}^{q_{2}+j-j^{\prime}} \frac{1}{D_{\epsilon}}\right)\right|_{v=\mu, s=f(\rho)} \sum_{|\alpha|=j^{\prime}} C_{\alpha} \mu^{\left(\alpha_{1}\right)} \cdots \mu^{\left(\alpha_{j^{\prime \prime}}\right)} \\
& +\left.\left(\partial_{s}^{p_{2}} \partial_{\rho}^{q_{2}+j} \frac{1}{D_{\epsilon}}\right)\right|_{v=\mu, s=f(\rho)} \\
\sim & \rho^{-(2 m+1)\left(q_{2}+j\right)} f(\rho)^{-p_{2}}
\end{aligned}
$$

for $q_{2}>0$ we have

$$
\left.\partial_{s}^{p} \partial_{\rho}^{q} \partial_{\tau}^{n^{\prime}} Q_{j}^{ \pm}(\tau, \rho ; s)\right|_{s=f(\rho)} \sim \rho^{-(2 m+1)(q+j+1)+1} f(\rho)^{-p-1} .
$$

It is desired estimate.

When we integrate derivatives of $Q_{j}^{ \pm}(\tau, \rho ; s)$ in $s$, a replacement of (6.16), which is not evaluated at $s=f(\rho)$, is needed.

Lemma 6.7. There exist continuous functions $L_{(p, q), k}(\rho ; s)$ on $\left(0, \rho_{0}\right) \times \mathbb{R}_{+}$, which are bounded as $\rho \rightarrow 0+$, such that

$$
\left.\left.\partial_{v}^{p} \partial_{\rho}^{q} \frac{1}{D_{\epsilon}(\rho ; s, v)}\right|_{v=\mu} \sim \rho^{-p-(2 m+1) q} \sum_{k=0}^{q} s^{p+k} f(\rho)^{-k} L_{(p, q), k}(\rho ; s) \frac{1}{D_{\epsilon}}\right|_{v=\mu} .
$$

Proof. By Lemma 6.1 and Lemma 6.3 we have

$$
\left.\frac{\partial_{v}^{p} \partial_{\rho}^{q} D_{\epsilon}}{D_{\epsilon}}\right|_{v=\mu} \sim \rho^{-p-q} \sum_{j=0}^{q} s^{p+j} f(\rho)^{-j} L_{p, j}(\rho ; s),
$$


where

$$
L_{p, j}(\rho ; s)= \begin{cases}\left.\hat{D}_{p, 0}\right|_{v=\mu} & j=0 \\ \left.\hat{D}_{p+j, 0}\right|_{v=\mu}+\left.\sum_{l=0}^{j-1} \rho^{-2 m(q-l)} \hat{D}_{p+l, j-l}\right|_{v=\mu} & j \geq 1\end{cases}
$$

Then

$$
\begin{aligned}
\left.\partial_{v}^{p} \partial_{\rho}^{q} \frac{1}{D_{\epsilon}}\right|_{v=\mu} & =\left.\sum_{i=1}^{\max (p, q)} \sum_{\substack{p_{1}+\cdots+p_{i}=p \\
q_{1}+\cdots+q_{i}=q}} \frac{\partial_{v}^{p_{1}} \partial_{\rho}^{q_{1}} D_{\epsilon}}{D_{\epsilon}} \cdots \frac{\partial_{v}^{p_{i}} \partial_{\rho}^{q_{i}} D_{\epsilon}}{D_{\epsilon}} \frac{1}{D_{\epsilon}}\right|_{v=\mu} \\
& \sim \sum_{k=0}^{q} \rho^{-p-(2 m+1) q} f(\rho)^{-k} s^{p+k} L_{(p, q), k}(\rho ; s),
\end{aligned}
$$

where

$$
\begin{aligned}
& \rho^{L_{(p, q), k}(\rho ; s)} \underset{\sum_{i=1}^{\max (p, q)}}{\operatorname{man}} \sum_{\substack{p_{1}+\cdots+p_{i}=p \\
q_{1}+\cdots+q_{i}=q}} \sum_{\substack{j_{1}+\cdots+j_{i}=k \\
0 \leq j_{1} \leq q_{1}, \ldots, 0 \leq j_{i} \leq q_{i}}} L_{p_{1}, j_{1}} \cdots L_{p_{i}, j_{i}} \\
\sim & \left(\sum_{i=1}^{\max (p, q)} \sum_{p_{1}+\cdots+p_{i}=p} \hat{D}_{p_{1}+j_{1}, 0} \cdots \hat{D}_{p_{i}+j_{i}, 0}+O(\rho)\right)
\end{aligned}
$$

as $\rho \rightarrow 0+$ and $\left|\hat{D}_{p_{1}+j_{1}, 0} \cdots \hat{D}_{p_{i}+j_{i}, 0}\right| \lesssim 1$. It completes the proof.

\section{References}

[1] G. Bharali, On the growth of the Bergman kernel near an infinite-type point, Math. Ann. 347 (2010), no. 1, 1-13.

[2] H. P. Boas, E. J. Straube, and J. Yu, Boundary limits of the Bergman kernel and metric, Michigan Math. J. 42 (1995), no. 3, 449-461.

[3] D. Catlin, Estimates of invariant metrics on pseudoconvex domains of dimension two, Math Z. 200 (1989), no. 3, 429-466.

[4] S. Cho, Boundary behavior of the Bergman kernel function on some pseudoconvex domains in $\mathbb{C}^{n}$, Trans. Amer. Math. Soc. 345 (1994), no. 2, 803-817.

[5] K. Diederich, Das Randverhalten der Bergmanschen Kernfunktion und Metrik in streng pseudo-konvexen Gebieten, Math. Ann. 187 (1970), 9-36.

[6] K. Diederich, G. Herbort, and T. Ohsawa, The Bergman kernel on uniformly extendable pseudoconvex domains, Math. Ann. 273 (1986), no. 3, 471-478.

[7] C. Fefferman, The Bergman kernel and biholomorphic mappings of pseudoconvex domains, Invent. Math. 26 (1974), 1-65.

[8] L. Hörmander, $L^{2}$ estimates and existence theorems for the $\bar{\partial}$ operator, Acta Math. 113 (1965), 89-152.

[9] J. Kamimoto, Asymptotic expansion of the Bergman kernel for weakly pseudoconvex tube domains in $\mathbb{C}^{2}$, Ann. Fac. Sci. Toulouse Math. (6) 7 (1998), no. 1, 51-85.

[10] Newton polyhedra and the Bergman kernel, Math. Z. 246 (2004), no. 3, 405-440.

[11] - The Bergman kernel on tube domain of finite type, J. Math. Sci. Univ. Tokyo 13 (2006), no. 3, 365-408. 
[12] K. Kim and S. Lee, Asymptotic behavior of the Bergman kernel and associated invariants in certain infinite type pseudoconvex domains, Forum Math. 14 (2002), no. 5, 775-795.

[13] A. Korányi, The Bergman kernel function for tubes over convex cones, Pacific J. Math. 12 (1962), no. 1, 1355-1359.

[14] J. McNeal, Estimates on the Bergman kernels of convex domains, Adv. Math. 109 (1994), 108-139.

[15] E. V. Vinberg, The theory of homogeneous convex cones, Trudy Moskov. Math. Obsc. 12 (1963), 303-358; Trans. Moscow Math. Soc. 12 (1963), 303-358.

Global Leadership School

Handong Global University

POHANG 791-708, KorEA

E-mail address: hxl@handong.edu 